University of Wollongong

Research Online

Faculty of Engineering and Information

Faculty of Engineering and Information

Sciences - Papers: Part A

Sciences

$1-1-2005$

Box-minus operation and application in sum-product algorithm

S Tong

Xidian University, sheng@uow.edu.au

P Wang

Xidian University

D Wang

Xidian University

X Wang

Xidian University

Follow this and additional works at: https://ro.uow.edu.au/eispapers

Part of the Engineering Commons, and the Science and Technology Studies Commons

Research Online is the open access institutional repository for the University of Wollongong. For further information contact the UOW Library: research-pubs@uow.edu.au 


\title{
Box-minus operation and application in sum-product algorithm
}

\begin{abstract}
A new formula for box-minus operation, which separates the box-minus operation into sign and reliability operations, was derived. Its application in the sum-product algorithm (SPA) was also investigated. Both box-plus operation and box-minus operation were divided into sign operation and reliability operation. The results show that on using the box-minus operation, SPA can be implemented with less decoding latency and complexity.
\end{abstract}

\section{Keywords}

product, algorithm, sum, application, operation, minus, box

Disciplines

Engineering | Science and Technology Studies

\section{Publication Details}

S. Tong, P. Wang, D. Wang \& X. Wang, "Box-minus operation and application in sum-product algorithm," Electronics Letters, vol. 41, (4) pp. 197-198, 2005. 


\section{Box-minus operation and application in sum-product algorithm}

\section{S. Tong, P. Wang, D. Wang and X. Wang}

\begin{abstract}
A new expression for box-minus operation, i.e. the inverse of box-plus operation, is derived, with which the box-minus operation can be implemented by a small look-up table. Its application in the sumproduct algorithm is investigated.
\end{abstract}

Introduction: For the practical application of low density parity check (LDPC) codes [1], the implementation of the associated decoding algorithm, i.e. the sum-product algorithm (SPA), should be carefully considered. As for this problem, there have already been some results [2-5]. An efficient parallel implementation in the log-likelihood ratio (LLR) domain for SPA is proposed in [4] leading to less decoding complexity and latency, which involves two core operations, i.e. boxplus operation [6] and its inverse, called box-minus operation.

In this Letter we derive a new expression for the box-minus operation, which separates the box-minus operation into sign and reliability operations and is more suitable for practical implementation.

Box-plus and box-minus operations: Denote the LLR of a binary random variable $u(\in\{ \pm 1\})$ as $L(u)=\log (P(u=+1) / P(u=-1))$. Then, for two independent binary random variables $u$ and $v$, the box-plus operation is defined as follows $[6,4]$ :

$$
\begin{aligned}
L(w)= & L(u \oplus v)=L(u) \boxplus L(v)=\log \frac{1+e^{L(u)} e^{L(v)}}{e^{L(u)}+e^{L(v)}} \\
= & \operatorname{sign}(L(u)) \operatorname{sign}(L(v)) \min \{|L(u)|,|L(v)|\} \\
& +\log \left(1+e^{-|L(u)+L(v)|}\right)-\log \left(1+e^{-|L(u)-L(v)|}\right)
\end{aligned}
$$

where $w=u \oplus v$ and $\oplus$ denotes binary XOR operation. Equation (1) can be further written as

$$
\begin{aligned}
L(w)= & L(u) \boxplus L(v)=\operatorname{sign}(L(u)) \operatorname{sign}(L(v)) \times\{\min \{|L(u)|,|L(v)|\} \\
& \left.+\log \left(1+e^{-(|L(u)|+|L(v)|)}\right)-\log \left(1+e^{-|| L(u)|-| L(v)||}\right)\right\}
\end{aligned}
$$

In (2), the term next to the multiplication sign is non-negative, and thus the box-plus operation is divided into sign and reliability operations. Then the box-plus operation reduces to the computation of the function $g(x)=\log \left(1+e^{-|x|}\right)$, which can be implemented by a small look-up table [4].

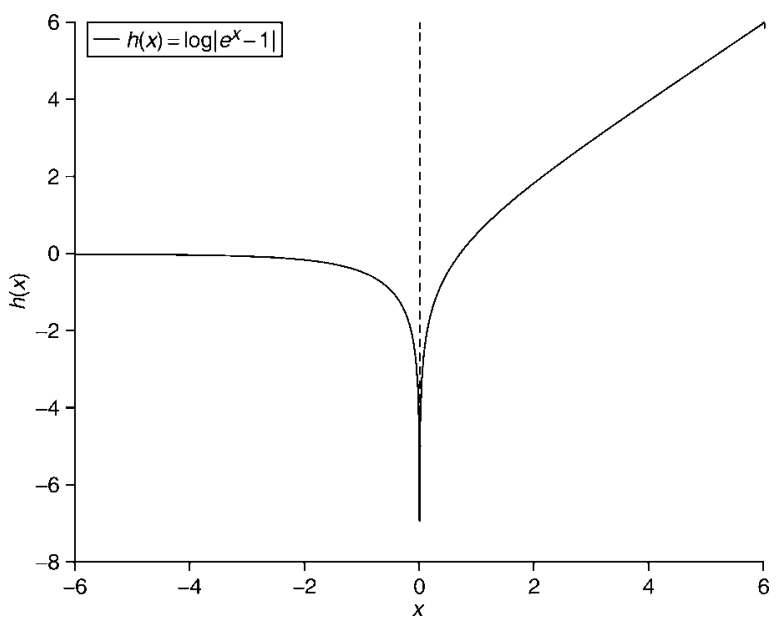

Fig. 1 Function $h(x)=\log \left|e^{x}-1\right|$

From (1), the box-minus operation can be defined as

$$
L(u)=L(w) \boxminus L(v)=\log \frac{1-e^{L(w)} e^{L(v)}}{e^{L(w)}-e^{L(v)}}
$$

Similar to the derivation of (2), the box-minus operation can be divided into sign and reliability operations as follows:

$$
\begin{aligned}
L(u)= & L(w) \boxminus L(v)=\operatorname{sign}(L(w)) \operatorname{sign}(L(v)) \times\{|L(w)| \\
& \left.+\log \left(1-e^{-(|L(w)|+|L(v)|)}\right)-\log \left(1-e^{-(|L(v)|-|L(w)|}\right)\right\}
\end{aligned}
$$

Since $|L(w)|<|L(v)|($ see (2)), the term next to the multiplication sign in the above expression is non-negative. Thus, the box-minus operation reduces to the function $\hat{h}(x)=\log \left(1-e^{x}\right),(x<0)$. A similar function $h(x)=\log \left|e^{x}-1\right|$ is defined in [4] for the implementation of box-minus operation, which is plotted in Fig. 1. It can be seen that as $x \rightarrow+\infty$, $h(x) \rightarrow+\infty$. Hence, $h(x)$ is not suitable to be implemented with a lookup table. However, $\hat{h}(x)$ is only the case of $h(x)$ when $x<0$, which can be easily implemented by a small look-up table.

Application of box-minus operation in SPA: Consider a check node $w$ of degree $n$. Denote its $n$ neighbouring variable nodes as $\left\{u_{k}\right\}_{k=1}^{n}$. In the horizontal step of SPA, the $n$ LLRs of

$$
\sum_{k \neq i} \oplus u_{k}, \quad(i=1,2, \ldots, n)
$$

are calculated [2-4]. Obviously, these LLRs can be calculated as follows:

$$
\begin{aligned}
L\left(\sum_{k \neq i} \oplus u_{k}\right) & =\sum_{k \neq i} \boxplus L\left(u_{k}\right)=L(w) \boxminus L\left(u_{i}\right) \\
L(w) & =L\left(\sum_{k=1}^{n} \oplus u_{k}\right)=\sum_{k=1}^{n} \boxplus L\left(u_{k}\right)
\end{aligned}
$$

In the conventional implementation, a forward-backward algorithm is used for the horizontal step, which involves $3(n-2)$ box-plus operations [4]. However, by first calculating $L(w)$ and then box-minusing $L\left(u_{i}\right)(i=1,2, \ldots, n)$, respectively, to get the $n$ LLRs, the overall computation is $(n-1)$ box-plus operations and $n$ box-minus operations. Since box-minus operation has almost the same computation complexity as box-plus operation, by the above technique the total computation complexity is reduced. In addition, the decoding latency is reduced from $\mathrm{O}(n)$ to $\mathrm{O}(\log (n))$ box-plus (or box-minus) operations [4]. Here we call this technique the parallel-excluding (PE) technique, which can be viewed as a message-passing schedule [7]. Denote the SPA implemented with PE as PE-SPA. Fig. 2 shows the bit error rate performance of a randomly constructed $(1008,3,6)$ regular LDPC code, assuming an AWGN channel and BPSK modulation. Here, the box-minus operation in PE-SPA is implemented by a 5-bit look-up table.

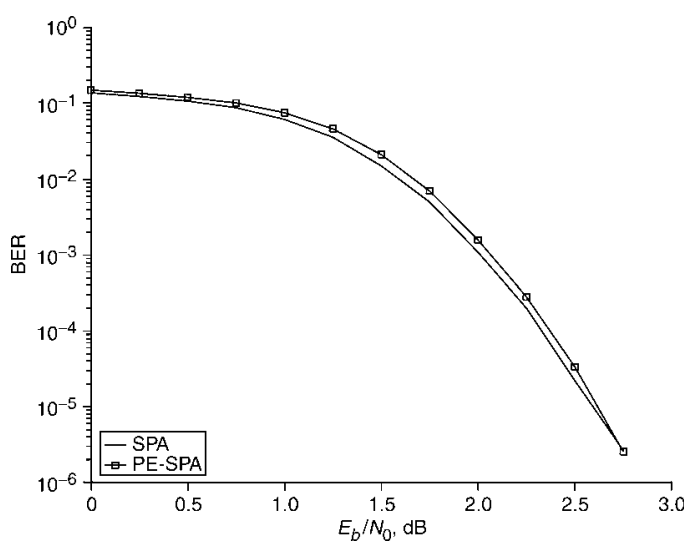

Fig. 2 Performance of $(1008,3,6)$ LDPC code

Conclusion: Both box-plus operation and box-minus operation are divided into sign operation and reliability operation. By its new expression, the box-minus operation can be implemented by a small look-up table. Using the box-minus operation, SPA can be implemented with less decoding latency and complexity.

(C) IEE 2005

17 October 2004

Electronics Letters online no: 20057447

doi: 10.1049/el:20057447

S. Tong, P. Wang, D. Wang and X. Wang (State Key Lab of ISN, Xidian University, Xi'an, 710071, People's Republic of China)

E-mail: ts_xd@163.com 


\section{References}

1 Gallager, R.G.: 'Low-density parity-check codes' (MIT Press, Cambridge, MA, 1963)

2 Ping, L., and Leung, W.K.: 'Decoding low density parity check codes with finite quantization bits', IEEE Commun. Lett., 2000, 4, pp. 62-64

3 Leung, W.K., Lee, W.L., Wu, A., and Ping, L.: 'An efficient implementation technique of LDPC decoder', Electron. Lett., 2001, 37, (20), pp. 1231-1232

$4 \mathrm{Hu}, \mathrm{X} .-$ Y., Eleftheriou, E., Amold, D.-M., and Dholakia, A.: 'Efficient implementation of the sum-product algorithm for decoding LDPC codes'. GLOBECOM, 2001
5 He, Y.-C., Sun, S.-H., and Wang, X.-M.: 'Fast decoding of LDPC codes using quantization', Electron. Lett., 2002, 38, (4), pp. 189-190

6 Hagenauer, J., Offer, E., and Papke, L.: 'Iterative decoding of binary block and convolutional codes', IEEE Trans. Inf. Theory, 1996, 42, (2), pp. $429-445$

7 Kschischang, F.R., and Frey, B.J.: 'Iterative decoding of compound codes by probability propagation in graphical models', IEEE J. Sel. Areas Commun., 1998, 16, (2), pp. 219-230 